\title{
Institucionalización del aprendizaje-servicio en una universidad intercongregacional
}

\section{Leticia López}

Universidad de Monterrey, México

\section{Resumen}

La Universidad de Monterrey (UDEM) se ha caracterizado desde su fundación como una universidad orientada a la mejora de las condiciones de vida de la comunidad, por medio del servicio y la acción social. Se empezó el proceso de implementación del aprendizaje-servicio (ApS) a nivel licenciatura durante el 2012 y actualmente es obligatorio. El objetivo de este trabajo es analizar las relaciones interdepartamentales durante el proceso de institucionalización del ApS, considerando sus principios fundacionales y visión intercongregacional desde una perspectiva sociológica para la generación de un modelo replicable en contextos educativos de inspiración católica. Se analizan las relaciones de los actores que contribuyeron a la institucionalización interna, desde la teoría de la estructuración de Anthony Giddens mediante el análisis de sus interacciones interdepartamentales. Este análisis permite crear un modelo de operación interna para impulsar el ApS de forma transversal en la institución. Ello permite comprender y generar conocimiento basado en prácticas sociales recurrentes.

\section{Palabras clave}

Institucionalización, aprendizaje-servicio, intercongregacional, estructura organizacional. 


\title{
Institutionalization of service-learning in an intercongregational university
}

\begin{abstract}
The University of Monterrey (UDEM) has been characterized since its foundation as a university aimed at improving the living conditions of the community, through service and social action. The process of implementation of service-learning (SL) began at the undergraduate level during 2012 and is currently mandatory. The objective of this work is to analyze interdepartmental relations during the process of institutionalization of the SL, considering its founding principles and intercongregational vision from a sociological perspective for the generation of a replicable model in Catholic-inspired educational contexts. The relationships of the actors that contributed to internal institutionalization are analyzed, from the structuring theory of Anthony Giddens through the analysis of their interdepartmental interactions. This analysis allows the creation of an internal operating model to promote SL across the institution. This allows understanding and generating knowledge based on recurring social practices.
\end{abstract}

\section{Key words}

Institutionalization, service-learning, intercongregational, organizational structure. 


\section{Institucionalització de l'aprenentatge servei en una universitat intercongregacional}

\section{Resum}

La Universidad de Monterrey (UDEM) s'ha caracteritzat des de la seva fundació com una universitat orientada a la millora de les condicions de vida de la comunitat, per mitjà del servei i l'acció social. Es va començar el procés d'implementació de l'aprenentatge servei (ApS) a nivell de llicenciatura durant el 2012 i actualment és obligatori. L'objectiu d'aquest treball és analitzar les relacions interdepartamentals durant el procés d'institucionalització de l'ApS, considerant els seus principis fundacionals i la visió intercongregacional des d'una perspectiva sociològica per a la generació d'un model replicable en contextos educatius d'inspiració catòlica. S'analitzen les relacions dels actors que van contribuir a la institucionalització interna, des de la teoria de l'estructuració d'Anthony Giddens mitjançant l'anàlisi de les interaccions interdepartamentals. Aquesta anàlisi permet crear un model d'operació interna per impulsar l'Aps de forma transversal a la institució. Això permet comprendre i generar coneixement basat en pràctiques socials recurrents.

\section{Paraules clau}

Institucionalització, aprenentatge servei, intercongregacional, estructura organitzacional. 


\section{Introducción}

La Universidad de Monterrey (UDEM) fue fundada el 8 de septiembre de 1969 por cinco congregaciones religiosas: las Hijas de María Inmaculada de Guadalupe, religiosas del Sagrado Corazón, Hermanas del Verbo Encarnado, los Hermanos Maristas y los hermanos Lasallistas. Se origina como seguimiento a la recomendación del Concilio Vaticano II de emplear las actividades educativas como medio de difusión de los principios católicos. Es decir, estas congregaciones religiosas impulsadas por ciudadanos católicos y empresarios de Nuevo León, dieron inicio a esta propuesta educativa que tiene tres principios fundacionales: humanismo, apertura y servicio.

Esta universidad se encuentra ubicada en la zona metropolitana de Monterrey (ZMM), que se encuentra caracterizada por tener indicadores de desarrollo superiores al de la mayoría de las ciudades de México a la par que presenta hogares en condiciones de pobreza similares a los observados en las regiones más pobres del país. Ejemplo de esta situación se refleja en que 2 de cada 5 personas son pobres, 2 más son vulnerables por sus bajos ingresos, y solo 1 no es ni pobre ni vulnerable. De acuerdo con la última medición de pobreza en el país, se registraron 52.4 millones de personas pobres y 9.3 millones de ellas vivían en pobreza extrema (Consejo Nacional de Evaluación de la Política de Desarrollo Social [CONEVAL], 2018).

La UDEM se ha caracterizado desde su

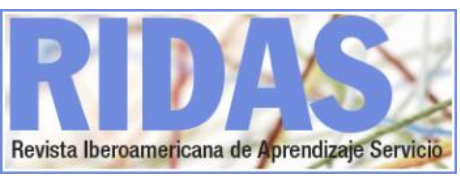

fundación como una universidad orientada a la mejora de las condiciones de vida de la comunidad donde opera, por medio del servicio y la acción social, "El ser humano solo alcanza su plenitud en el servicio a los demás" (UDEM, 2021). Consecuencia de esta orientación humanista ha sido la creación de proyectos institucionales orientados a contribuir a la reducción de las condiciones de vulnerabilidad y pobreza en que se encuentran sus comunidades aledañas desde una perspectiva académica, por ejemplo los proyectos Universidad de Barrios, KIMAKUL, Centros de Salud y Desarrollo, Reconstrucción del Tejido Social, Preparatorias Politécnicas, Formar para Transformar, etc. (UDEM, 2020). Proyectos que han tenido como objetivo el desarrollo de una conciencia y responsabilidad social en los estudiantes, con la finalidad de generar una transformación social como respuesta a las problemáticas sociales, económicas y ambientales del país.

\section{Metodología}

La metodología utilizada para analizar las relaciones entre los diferentes departamentos fue a través de entrevistas para identificar la historia, así como los retos y dinámicas sociales de las interacciones durante el proceso de institucionalización. Se establecieron períodos de tiempo y actores diversos que fueron parte de esas etapas durante el proceso. Se realizó una categorización de los actores entre académicos y de formación integral, los primeros relacionados con las disciplinas de conocimiento de los diferentes programas que la UDEM ha

López, L. (2021). Institucionalización del aprendizaje-servicio en una universidad intercongregacional. RIDAS, Revista Iberoamericana de Aprendizaje Servicio, 12, 24-34. DOI10.1344/RIDAS2021.12.4 
ofrecido a través del tiempo y los segundos hacia aquellos enfocados a la formación extracurricular de los estudiantes que es donde surgió el aprendizaje-servicio. El análisis es mediante la Teoría de la Estructuración para entender esas interacciones y desde los carismas fundantes de cada una de las cinco congregaciones fundadoras.

Se entrevistaron 10 personas categorizadas en tiempo y área de enfoque para comprender la línea del tiempo del proceso y las interacciones que sucedieron para lograrlo. Los hallazgos desde el marco de sus carismas fundantes, debido a que la UDEM es considerada como heredera de la espiritualidad y los carismas de cinco congregaciones fundadoras ya tiene una madurez y debe imprimir un sello de espiritualidad, una mística muy particular, a la educación que ofrece a sus alumnos. En este trabajo de investigación se analizarán las acciones y relaciones de los actores colectivos e individuales que pertenecen a una determinada práctica social dentro del proceso de institucionalización y a una estructura organizacional dentro de la UDEM. No se pretende una metodología de análisis del discurso, pero si una comprensión de éste a través de las redes de relaciones y los documentos captados durante las múltiples interacciones de los actores estudiados.

\section{Resultados y discusión}

Se empezó el proceso de implementación del aprendizaje-servicio a nivel licenciatura durante el 2012, con la capacitación de 40 profesores de la universidad por parte del Centro
Latinoamericano de Aprendizaje y Servicio Solidario (CLAYSS). El acompañamiento a estos profesores se consolidó con la creación del área de aprendizaje-servicio dentro del Centro para la Solidaridad y la Filantropía (CESYF), el cual se encuentra en la Vicerrectoría de Formación Integral.

Posteriormente en el 2013, las Preparatorias de la UDEM integraron el aprendizaje-servicio como eje central de la materia de "Titularidad" durante el $3^{\circ}, 4^{\circ}, 5^{\circ}$ y $6^{\circ}$ semestre. En ese espacio, el profesor titular se convierte en el asesor porque acompaña el desarrollo y realización de los proyectos de los estudiantes, esta materia es cursada por el $100 \%$ de los estudiantes. Durante el 3r semestre trabajan en un proyecto social de forma individual, después en el $4^{\circ}$ semestre el proyecto es con todo el grupo, con la finalidad de prepararlos a lo que será el proyecto concreto de aprendizajeservicio que realizarán por un año en equipos y será evaluado para su acreditación a finales del $6^{\circ}$ semestre como requisito de graduación. El último año trabajan con una sola organización, desde el diagnóstico hasta la evaluación de la implementación de este. A las personas que son parte de las organizaciones de la sociedad civil se les Ilama supervisores porque evalúan el impacto del proyecto y finalmente los estudiantes reciben apoyo del coordinador de acciones solidarias que se encuentra en cada unidad de Preparatoria.

En el 2014, como consecuencia de los resultados que la implementación de la metodología tenía en estudiantes, profesores y

López, L. (2021). Institucionalización del aprendizaje-servicio en una universidad intercongregacional. RIDAS, Revista Iberoamericana de Aprendizaje Servicio, 12, 24-34. DOI10.1344/RIDAS2021.12.4 
comunidades/organizaciones, y con el interés de impulsar la consolidación de esta metodología de aprendizaje, se realiza el primer reconocimiento a profesores que la implementaron. Este reconocimiento fue entregado por el Vicerrector Académico con el objetivo de evidenciar el valor que esta metodología generaba desde los contenidos curriculares.

Durante el 2015, el CESYF en colaboración con el Centro de Innovación y Desarrollo (CID), diseñó el curso de aprendizaje-servicio para ser impartido dentro de la UDEM. El objetivo era brindar al profesorado el conocimiento y las herramientas necesarias para una adecuada adaptación e implementación de la estrategia, dándoles también un espacio para el rediseño de su materia. Con la creación de este curso, se logra que la metodología sea considerada como uno de los cinco métodos de aprendizaje que se contemplan dentro del Modelo Pedagógico de la Universidad. El $100 \%$ de los profesores de profesional de la universidad deben de certificarse en este modelo, pues en él se define experiencia de aprendizaje del estudiante y el perfil humanista que se desea a su egreso.

Durante el 2016, la Vicerrectoría de Formación Integral inició la implementación de una sesión en el Seminario de Inducción a la Carrera que cursan todos los estudiantes de primer ingreso en donde se les da a conocer lo que es aprendizaje-servicio y cómo pueden promoverlo entre sus profesores.

En este mismo año, se da un impulso importante a la implementación del aprendizaje-servicio en la UDEM al ser incluida dentro del Programa de la Mejora de la Calidad (QEP, por sus siglas en inglés) como la metodología a través de la cual se logrará uno de los tres resultados de aprendizaje del estudiante. Este programa establece el compromiso que adquiere la universidad ante la Southern Association of Colleges and Schools (SACS COC), una de las principales acreditaciones con las que cuenta la UDEM, para mejorar la calidad en el aprendizaje del estudiante. La inclusión del aprendizaje-servicio como metodología clave en la obtención de esta acreditación incentivó a que las Vicerrectorías Académicas promovieran su proceso de institucionalización de una forma más fuerte al interior de la universidad y con un mayor número de recursos humanos, materiales y económicos para su consolidación.

En el marco de este proceso de acreditación, en agosto del 2016, se forma el Comité QEP de aprendizajeservicio, el cual está integrado por representantes de cada una de las seis escuelas académicas, Centro de Innovación y Desarrollo y del Centro para la Solidaridad y la Filantropía. Este comité forma parte del Comité Ejecutivo del QEP, el cual reporta directamente al rector de la universidad.

Este Comité tiene dos objetivos: primero, que al menos dos cursos de cada programa académico sean impartidos bajo la metodología de aprendizaje-servicio; segundo, que todos los estudiantes de la UDEM implementen un proyecto de mejora

López, L. (2021). Institucionalización del aprendizaje-servicio en una universidad intercongregacional. RIDAS, Revista Iberoamericana de Aprendizaje Servicio, 12, 24-34. DOI10.1344/RIDAS2021.12.4 
comunitaria o del entorno, en donde apliquen los conocimientos y habilidades de la materia. Los cuales se están cumpliendo actualmente.

En el 2020 se creó un proceso de vinculación social virtual, para acompañar a los profesores en encontrar necesidades de las organizaciones de la sociedad civil que puedan ser atendidas por sus materias a través de aprendizaje-servicio a través de una plataforma tecnológico que permite unir necesidades en la comunidad y oferta académica.

Actualmente se ha continuado el impulso del aprendizaje-servicio dentro la UDEM en sus dos niveles (preparatoria y licenciatura), con el interés de incrementar el valor que tanto estudiantes como profesores pueden aportar a las comunidades y organizaciones por medio de los conocimientos adquiridos en sus materias académicas y cocurriculares. El proceso de institucionalización en que se encuentra la Universidad presenta varios retos, quizá el más grande es continuar la sensibilización de autoridades, profesores y estudiantes, para dar a conocer el potencial que tiene esta metodología para construir una mejor sociedad de forma directa y concreta, y evitar que sea vista como un requisito que se tiene que cumplir para lograr una acreditación o como un requisito de graduación. Otro reto es generar más incentivos que motiven su implementación, más allá de la obligatoriedad que actualmente tiene al interior de la Universidad y crear una comunidad donde la innovación social y el aprendizaje fuera del aula sean los elementos que generen sentido de pertenencia en el cuerpo docente que la implementa.

Después de 9 años de impulsar el aprendizaje-servicio dentro de la UDEM, se ha logrado que más de 8,000 estudiantes hayan tomado al menos un curso bajo esta metodología, a través de 870 proyectos y 117 profesores, mismos que han logrado beneficiar a 330 organizaciones de la sociedad civil. Asimismo, el proceso se ha documentado en reportes anuales y se han hecho 12 contribuciones académicas en diferentes revistas científicas y se realiza de forma anual el Seminario de Aprendizaje en el Servicio Universitario (SASU) con la finalidad de ser un espacio de intercambio de experiencias y aprendizajes al interior de la universidad entre profesores, estudiantes y directivos en donde se realiza también un premio al mejor proyecto de investigación relacionado con la implementación de aprendizajeservicio.

La suma de esfuerzos de diferentes áreas comprometidas con el desarrollo de la sociedad y las discusiones que se presentan en los diferentes espacios universitarios donde se realizan proyectos de aprendizaje-servicio, nos dan elementos para suponer que se logrará cumplir con la razón de ser de este metodología y de la UDEM: atender a las problemáticas que enfrenta nuestra sociedad por medio de conocimientos y habilidades al servicio de la comunidad, desde una perspectiva integral, ética y de corresponsabilidad con nosotros mismos y las futuras generaciones.

López, L. (2021). Institucionalización del aprendizaje-servicio en una universidad intercongregacional. RIDAS, Revista Iberoamericana de Aprendizaje Servicio, 12, 24-34. DOI10.1344/RIDAS2021.12.4 
En este contexto, el docente era un actor activo, profundamente involucrado en el proceso, convirtiéndose en un agente (Giddens, 2006), definido de la siguiente manera:

1. Un agente es competente: el criterio fundamental de competencia es que un actor es capaz de explicar todas las actividades que realiza, si se le pregunta. Y tenía claro sus motivaciones y trascendencia en términos de enseñanzaaprendizaje de lo que la metodología ofrecía en el aula.

2. El agente es reflexivo: la reflexividad es la capacidad del agente para registrar sistemáticamente aspectos sociales de los contextos en los que se mueve. Esta reflexividad es lo que posibilita las prácticas sociales recursivas. Esto se generaba de forma orgánica por los proyectos sociales fuera del aula a favor del bien común en conjunto con organizaciones de la sociedad civil.

3. El agente es intencional: el agente responde a las razones y motivos de sus acciones, aunque muchas de estas motivaciones no son parte de sus discursos. Giddens distingue dos elementos (ambos forman parte de un modelo): racionalización y motivación. La racionalización corresponde a lo teórico, y la motivación lo que le mueve a actuar, desde deseos que no son necesariamente conocidos por el agente. $\mathrm{Y}$ los actores entrevistaron mostraron una vocación por el servicio previamente de dedicarse a la docencia o a la implementación de la metodología de aprendizaje-servicio en el aula, es decir, existe un componente de propósito de vida y otro relacionado con su función docente, que se mezclan.

Así que el principal agente que permitió que el proceso de institucionalización avanzará fue el docente, independientemente de los cambios en la estructura organizaciones para que esto se lograra. Y esto empezó antes de que ingresará el concepto de aprendizaje-servicio en la UDEM, porque está en la esencia desde su fundación cuando existía un panorama particular que vivía México durante 1968 y cómo ante todo aquello surge en un grupo de religiosos y laicos la necesidad de emprender una universidad diferente, una institución en la cual el hombre y su desarrollo fueran el centro del quehacer universitario y el servicio a los más necesitados fuera el medio para la trascendencia. Siendo el carisma de cada congregación fundadora las bases de su creación, considerando que es una especial capacidad de algunas personas para atraer o fascinar. Desde el punto de vista religioso el carisma lo define como un don gratuito que Dios concede a las personas en beneficio de la comunidad.

La novedad de la UDEM es la integración de un todo armónico y funcional de elementos comunes de las cinco Congregaciones Religiosas que abiertas a la idea de esta unificación 
decidieron acometerse en su relación conjuntamente con un gran número de seglares y con la anuencia y bendición del Arzobispo de Monterrey. Esto equivale a una pedagogía sobre la fe como dice la cita "...sean todos uno... para que el mundo crea" (Biblia online, 2021, Juan, 17: 21). Y el aprendizajeservicio permitió hacer vida lo anterior.

\section{Conclusiones}

Los Principios de la UDEM se asientan en el Convenio Intercongregacional que firmaron las cinco congregaciones fundadoras el 30 de enero de 1972. Dicho convenio recibió la bendición del Sr. Arzobispo de la Ciudad de Monterrey, Ilmo. Sr. Don Alfonso Espino y Silva y de los Superiores Mayores de cada una de las congregaciones participantes y fundadores.

Es una Universidad abierta al mundo para recibir de él los valores y la cultura; a todos los hombres para transmitir estos valores. Es abierta porque pretende contribuir con hechos al diálogo con la cultura y a servir desinteresadamente al hombre, poniendo en práctica los enfoques y métodos que sean positiva aportación a la Reforma Educativa.

Considera a la persona humana como el origen y centro de la cultura; por eso sitúa al joven universitario como hombre, cualquiera sea su origen, o condición familiar, social y así le da un sentido a la vida; lo sitúa como un ser en relación, procurando que todo universitario entre sí, y universitarios y maestros, formen la comunidad educativa, donde sea posible el aprendizaje de la libertad, del diálogo, del autogobierno.

Es una Universidad para el servicio. Sí, a los fines tradicionales y esenciales de cualquier universidad: Conservación, transmisión y progreso de la cultura, preparación de profesionistas, formación integral humana, investigación científica, la UDEM podría agregar como característica suya y muy especial: una Universidad para el servicio, que propiciará las prácticas de campo con espíritu no de adquirir sólo habilidad ocupacional, sino de servir. $Y$ como se dijo anteriormente, se alentará a los universitarios a través de una mística del servicio, a insertarse activamente en las agrupaciones que constituyen la colectividad humana y a que colaboren en los esfuerzos para promover el progreso social y el desarrollo especialmente en México.

Durante el proceso de institucionalización del aprendizajeservicio se permeaban estos principios establecidos por las diferentes congregaciones fundadoras y en las relaciones entre los actores a través del tiempo, contribuyeron al proceso, que desencadenó varios tipos de socialización, que se relacionan con las posturas de Gratnovetter (1985), Melluci (1999) y AbouAssi (2013) como disruptivo, articulador resiliente e interdependiente:

\section{Agentes Protectores}

(interdependientes): son los responsables de asegurar la implementación del aprendizajeservicio desde una postura institucional considerando sus principios y valores fundacionales. Son los 
departamentos académicos y las áreas de formación integrar responsables de administrar las materias y acompañar docentes. $Y$ que requieren interactuar con diferentes áreas tanto administrativas como académicas y de comunicación internas para que todo el proceso se realice.

2. Agentes Reguladores (disruptivos): han sido las áreas responsables de hacer vida el aprendizaje-servicio desde una estructura organizacional que evoluciona y cambia con el tiempo, como lo fue el Centro para la Solidaridad y la Filantropía y posteriormente el Comité del Plan de Mejoramiento de la Calidad del Aprendizaje con representantes de cada Escuela Académica para implementar nuevos procesos y políticas que permitan que las decisiones tomadas a nivel institucional de convertir el aprendizaje-servicio en obligatorio se pueda lograr. Estos agentes se enfrentaron a resistencias al cambio que permitió contribuir a la generación del marco normativo actual que define como debe operar e implementarse el aprendizaje-servicio en la UDEM.

3. Agentes Cuidadores (articuladores resilientes): son los docentes que han requerido aprender con la practica a estas nuevas formas de enseñanza e integrando nuevos actores como las organizaciones de la sociedad civil, gobierno en diversos contextos sociales en situación de vulnerabilidad que generó la necesidad de desarrollar capacidades para administrar alianzas y convenios a favor del bien común por los compromisos adquiridos a través de sus materias con realidades concretas.

\section{Agradecimientos}

Agradezco a cada uno de los actores entrevistados, docentes, miembros del Comité del Plan de Mejoramiento de la Calidad del Aprendizaje, colegas del Centro para la Solidaridad y Filantropía, así como la Dirección de Identidad y Principios Institucionales.

\section{Referencias bibliográficas}

AbouAssi, K. (2013). Integrating resource dependency theory and theory of weak ties to understand organizational behavior. Madison: University of Wisconsin.

Biblia online. (2021). El Libro del Pueblo de Dios. Editorial San Pablo.

https://www.sanpablo.es/libro-pueblode-dios/la-biblia/nuevo-

testamento/evangelio-segun-sanjuan/17

Consejo Nacional de Evaluación de la Política de Desarrollo Social. (2018). Medición de la pobreza 2018. https://www.coneval.org.mx/Medicion/ MP/Paginas/Pobreza-2018.aspx

Giddens, A. (2006). Sociology (5th ed). Polity.

Gratnovetter, M.S. (1985). Economic action and social structure: the problem 
of embeddedness. American Journal of Sociology, 91(3), 481-510.

Mellucci, A. (1999). Acción colectiva, vida cotidiana y democracia. Colegio de México - Centro de Estudios

Sociológicos.

Universidad de Monterrey [UDEM].

(2021). Principios fundacionales.

https://www.udem.edu.mx/es/conoce/p

$\underline{\text { rincipios-fundacionales }}$

Universidad de Monterrey [UDEM].

(2020). Formación de la conciencia y acción social.

https://www.udem.edu.mx/es/vive/for

macion-de-la-conciencia-y-accion-social 